\title{
Diseño teórico de la investigación: instrucciones metodológicas para el desarrollo de propuestas y proyectos de investigación científica
}

\author{
José R. García-González ${ }^{1 *}$ y Paola A. Sánchez-Sánchez ${ }^{2}$ \\ (1) Facultad de Ingenierías, Universidad Simón Bolívar, Barranquilla, Colombia. ORCID: 0000-0002-3365-6095 \\ (correo-e: jgarcia122@unisimonbolivar.edu.co) \\ (2) Facultad de Ingenierías, Universidad Simón Bolívar, Barranquilla, Colombia. ORCID: 0000-0002-3320-016X \\ (correo-e: psanchez9@unisimonbolivar.edu.co)
}

Recibido Jun. 23, 2020; Aceptado Ago. 21, 2020; Versión final Sep. 8, 2020, Publicado Dic. 2020

\begin{abstract}
Resumen
En este estudio se propone un esquema de integración sistémica entre los diferentes elementos del diseño teórico de la investigación desde los principios rectores de la investigación holística. Se define una metodología de trabajo orientada a la identificación de los elementos clave del proceso investigativo a partir del análisis sistémico de la situación problémica y su relación con el objeto de estudio y su campo de acción. Se incluye la definición de categorías rectoras, la integración sistémica de contenidos y la presentación de un esquema sintético para la orientación metodológica del proceso investigativo. Como resultado, se presenta la sistematización epistemológica del proceso investigativo a través de dos casos aplicativos. Se concluye que el esquema orientador presentado permite generar una didáctica propia para orientar el desarrollo de propuestas y proyectos de investigación científica de calidad, alto impacto y pertinencia, y favorece la comprensión de los procesos y cursos de investigación científica en la educación superior.
\end{abstract}

Palabras clave: metodología de la investigación; investigación científica; investigación holística; proyectos de investigación

\section{Theoretical design of research: methodological instructions for the development of scientific research proposals and projects}

\begin{abstract}
The present study proposes a systemic integration scheme for theoretical design research elements by examining holistic research guiding principles. A working methodology is defined that is aimed at identifying key elements in the research process. This is based on the systemic analysis of every problem and its relationship with the object of study and the field of action. This includes the definition of guiding categories, the systemic integration of contents, and the presentation of a synthetic scheme for the methodological orientation of the research process. As a result, the epistemological systematization of the research process is presented in two applied cases. It is concluded that the guiding scheme presented allows generating didactic tools that can guide the development of high quality, high impact, and relevant scientific research proposals and projects. In addition, the guiding scheme favors the understanding of processes and courses in higher education scientific research.
\end{abstract}

Keywords: research methodology; scientific research; holistic research; research projects 


\section{INTRODUCCIÓN}

La investigación a través de los años se ha asociado con la aplicabilidad del método científico en procesos en los cuales se requiere obtener información, para entender, verificar, corregir o aplicar el conocimiento en un determinado escenario (González y Hernández, 2014; Gómez, 2019). De igual forma, para poder obtener de manera clara y precisa un resultado, se hace necesario al menos la aplicación de un tipo de investigación, la cual provee una serie de elementos y pasos que son necesarios para alcanzar el objetivo propuesto o para poder obtener la información que se requiere con base en el análisis de una serie necesidades que desde lo social, deberán identificarse (Marczyk, et al., 2005; Corona, 2018; Ortíz, 2012; Davis, et al., 2014).

El proceso de investigación se soporta en el método científico, definido por muchos autores como el método de estudio sistemático de la naturaleza, el cual incluye: técnicas de observación, reglas para el razonamiento y la predicción, ideas sobre y para la experimentación planificada y las diferentes formas para la comunicación y divulgación de los resultados obtenidos desde lo experimental como desde lo teórico. Existe una manera de hacer las cosas, de plantearse preguntas y de formular respuestas, acciones que son características de la ciencia, lo cual permite de manera lógica a quien investiga, desarrollar un trabajo con orden y racionalidad (Grant y Booth, 2009; Snyder, 2019).

La metodología de la investigación suele asumirse por muchos como un "ladrillo", expresión coloquial que en el entorno académico colombiano significa algo de difícil entender, algo riguroso, incómodo y en ocasiones tortuoso de tratar y manejar por quienes no han tenido formación y/o experiencia en el campo de la investigación científica. Así las cosas, no es secreto que los cursos, asignaturas, seminarios, diplomados, conferencias o cualquier otro tipo de actividad relacionada con la enseñanza - aprendizaje o aplicabilidad de la metodología de la investigación científica, se asumen con recelo y cierto nivel de escepticismo por parte de estudiantes, profesores e incluso investigadores; lo anterior se suscita debido a que aún se sigue insistiendo en la tradicionalidad en los procesos de enseñanza - aprendizaje de este tipo de conocimientos y a la rigurosidad de las didácticas empleadas para tales fines (Ramírez y Zwerg-Villegas, 2012; Mackenzie y Knipe, 2006; Génova y González, 2012).

A pesar de mantenerse la tradicionalidad del método científico sobre todo en procesos de investigación aplicada y del orden técnico, este método, asumido como de investigación, admite las siguientes críticas: primero, la rigurosidad de su aplicación no admite siempre una ruta fija o predeterminada o un algoritmo con una serie de instrucciones inmodificables en un mismo ciclo; y segundo, el conocimiento científico no se adquiere a partir de un proceso similar, sino que se va desarrollando gracias a la libertad e independencia de pensamiento, mediante la crítica, el análisis riguroso, la superación de los errores y la discusión. Lo anterior presupone que la aplicabilidad rigurosa del método no garantiza el éxito del proceso, como tampoco podría garantizarse el fracaso del mismo (Bayazit, 2004; Haddadi et al., 2017; Schmidt et al., 2019).

Históricamente se han desarrollado y definido diversas concepciones del proceso investigativo o modelos epistémicos de apoyo al mismo, con la intensión de poder estructurar nuevos procesos que favorezcan y faciliten la labor investigativa en todos los niveles de formación; la gran mayoría de dichos modelos se encuentran identificados y encuadrados en el paradigma cualitativo de investigación, de acuerdo con los estudios de Schmitt (2005), Thomas y Harden (2018) y Saunders y Bezzina (2015). Los anteriormente citados y otros autores relevantes, coinciden en la necesidad de proponer una manera diferente de hacer investigación, una manera más simple, ajustada y consecuente con los múltiples enfoques emergentes producto de los aportes de la llamada sociedad de la información y del conocimiento. Sin embargo, las nuevas propuestas suelen estar más asociadas con lo fenomenológico y en muchas ocasiones son asociadas a los procesos y al ser humano, lo que para algunos signifique una ruptura entre lo cualitativo y lo cuantitativo, entrando entonces en una discusión epistémica al respecto de lo opuestos o complementarios que pudieren ser estos dos enfoques (Corona, 2018).

De igual manera, las discusiones teóricas y epistemológicas respecto a los diferentes paradigmas, entendiéndose estos como los logros científicos que generan modelos que, durante un período más o menos largo, y de modo más o menos explícito, orientan el desarrollo posterior de las investigaciones exclusivamente en la búsqueda de soluciones para los problemas planteados por estas, pudieran ser un tanto imprecisas, puesto que las denominaciones "cualitativo" y "cuantitativo" que de acuerdo con Hurtado (2014), harían referencia a las diferentes formas de simbolizar o codificar la información y asociadas de manera específica con las técnicas de recolección de datos y de análisis de los mismos, pero no a modelos epistémicos definidos; lo último hace referencia a que, en la denominación de paradigma cualitativo, se cobijan diferentes modelos epistémicos (estructuralismo, pragmatismo, materialismo histórico dialéctico, entre otros) que difieren mucho entre sí con respecto a su noción de conocimiento, del ser humano, de la sociedad, entre otros aspectos. De igual manera, el proceso investigativo contiene ambos aspectos (cualitativo y cuantitativo), pues se trata de formas complementarias de codificar y procesar la información, las cuales permiten acceder a diferentes aspectos o manifestaciones de un mismo evento (Papachristos, 2018). 
En consecuencia, muchos teóricos de tendencia positivista (signados por el paradigma cuantitativo) aún defienden la teoría de que el único tipo de investigación válido y científico es la investigación confirmatoria de verificación empírica, la cual se soporta en el ya comentado método científico, no obstante, al dejarse de lado las modalidades de investigación no confirmatorias, ha desestimado vertientes importantísimas del quehacer investigativo como la investigación evaluativa, la investigación-acción, la proyectiva, la predictiva, la prospectiva, entre otras (Hurtado, 2014; Mertens, 2010).

Es válido argumentar que desde una comprensión holística, un nuevo paradigma, una nueva forma de pensar la investigación, una nueva manera de estructurar el proceso para hacerlo entendible, fácil de aplicar, no sería contradictorio con lo planteado hasta el momento, por el contrario, complementa lo descrito desde una perspectiva novedosa, original y sistémica, por tanto los diferentes modelos epistémicos (empirismo, estructuralismo, positivismo, pragmatismo, materialismo dialéctico, etc...), que de alguna manera se encuentran solapados bajo las denominaciones de paradigma cualitativo" y "cuantitativo, se consideran formas complementarias de percibir la misma realidad. Por lo tanto, la investigación holística se considera como una propuesta metodológica que expone el proceso investigativo como una totalidad organizada con carácter sistémico, dinámico e integrador. Este tipo de enfoque se sustenta en la incorporación de procesos simples y estructurados tendientes a la formulación y presentación de proyectos de investigación ajustados a las necesidades actuales y sobre todo haciendo uso de los nuevos modelos y herramientas que ofrecen las nuevas tecnologías, favoreciendo de esta manera cambios significativos en la labor investigativa asociados con la aplicabilidad de una metodología estructurada y con una didáctica propia que favorece el proceso de formación de investigadores.

En este artículo se explica la investigación científica como un proceso orientado a conocer, interpretar y comprender una realidad, que dentro de las necesidades del investigador se pueda intervenir con el objeto de poder lograr su transformación. El objetivo del artículo es presentar una metodología sistémica de trabajo desde una perspectiva holística que favorezca entender una situación que requiere ser atendida desde la aplicación de un método de ciencia, identificar su objeto de estudio y a partir de la integración de los mismos, poder identificar el mejor camino hacia la mejor alternativa de solución de dicha situación. La originalidad, significancia y relevancia de este trabajo está basada en los siguientes aspectos: 1) Se propone un esquema dinámico con carácter de sistema en el que se definen los diferentes elementos componentes del diseño teórico de la investigación y su interacción; 2) El carácter de sistema del esquema propuesto, sugiere una integración didáctica entre cada uno de los elementos componentes del diseño teórico de la investigación a partir de la lógica y características propias del proceso de investigación científica; 3) Se considera como una estructura metodológica que favorece la organización del proceso de investigación en todos los niveles con el objetivo de poder definir cada uno de los elementos necesarios para una investigación completa y ajustada con las necesidades que el investigador define en su proceso; 4) El enfoque holístico con el que se concibe el diseño teórico de la investigación favorece de forma sencilla el desarrollo de propuestas de investigación altamente exitosas con aplicación e implicación en diferentes ramas del saber científico.

\section{METODOLOGÍA}

La investigación científica como método, podría entenderse como un proceso sistémico que permite conocer una realidad objeto de estudio, sus características y condiciones en un entorno específico desde el plano teórico, práctico y/o, teórico - práctico, partiendo de la necesidad del investigador, a partir de la aplicación de conocimiento científico, poder abordar problemas sociales que requieren ser tratados como problemas de ciencia para entonces poder encontrar soluciones acordes y pertinentes con la realidad en la que el investigador se identifica (Davis et al., 2014).

El proceso de investigación científica emerge a partir de las necesidades sociales de dar solución a problemas rutinarios y de tratar de explicar diferentes fenómenos que permitan tener un mejor escenario de actuación frente a los distintos entornos de desarrollo del ser humano, lo que le imprime un carácter creativo e innovador desde el punto de vista científico (Caillaud et al., 2016). Es por lo anterior que definir un diseño teórico de investigación (DTI) deberá ser el primer paso para la construcción de un proyecto de investigación y sus productos científicos derivados. Existen razones suficientes para comprender que los componentes del diseño teórico de la investigación no deben ser acomodados o ajustados a procesos distintos a la investigación, sin embargo, su lógica y dinámica permiten ser aplicable a cualquier investigación en distintas áreas del conocimiento (por ejemplo, García et al., 2019; Sánchez-Sánchez et al., 2019). En consecuencia, los elementos componentes del diseño teórico son en definitiva quienes definen la estructura paradigmática de la investigación, dicho de otra forma, es el diseño teórico quien determina el futuro de la profundidad de un paradigma con respecto a otro.

La estrategia propuesta en este trabajo se basa en un enfoque sistémico y sistemático orientado a la especificación del diseño teórico de la investigación, destacando la importancia de considerar de forma sintética aspectos clave que permiten y conducen a una identificación clara de la propuesta o proyecto de 
investigación. A partir de la existencia de una idea de investigación, el esquema propuesto para la especificación del diseño teórico de la investigación (DTI) se presentan en la Figura 1. A continuación, se describen los elementos e interacciones presentadas en la figura 1:

Delimitación de la realidad que el investigador se propone intervenir a partir de su investigación. permitirá al investigador, poder identificar el objeto de estudio sobre el cual se definirán sus cualidades, características, categorías rectoras, variables intervinientes y las relaciones entre cada una de ellas.

Situación que emerge de un conjunto complejo de factores (Situación problémica) que se articulan generando un escenario delimitado y concreto que amerita ser intervenido desde la aplicabilidad del conocimiento científico.
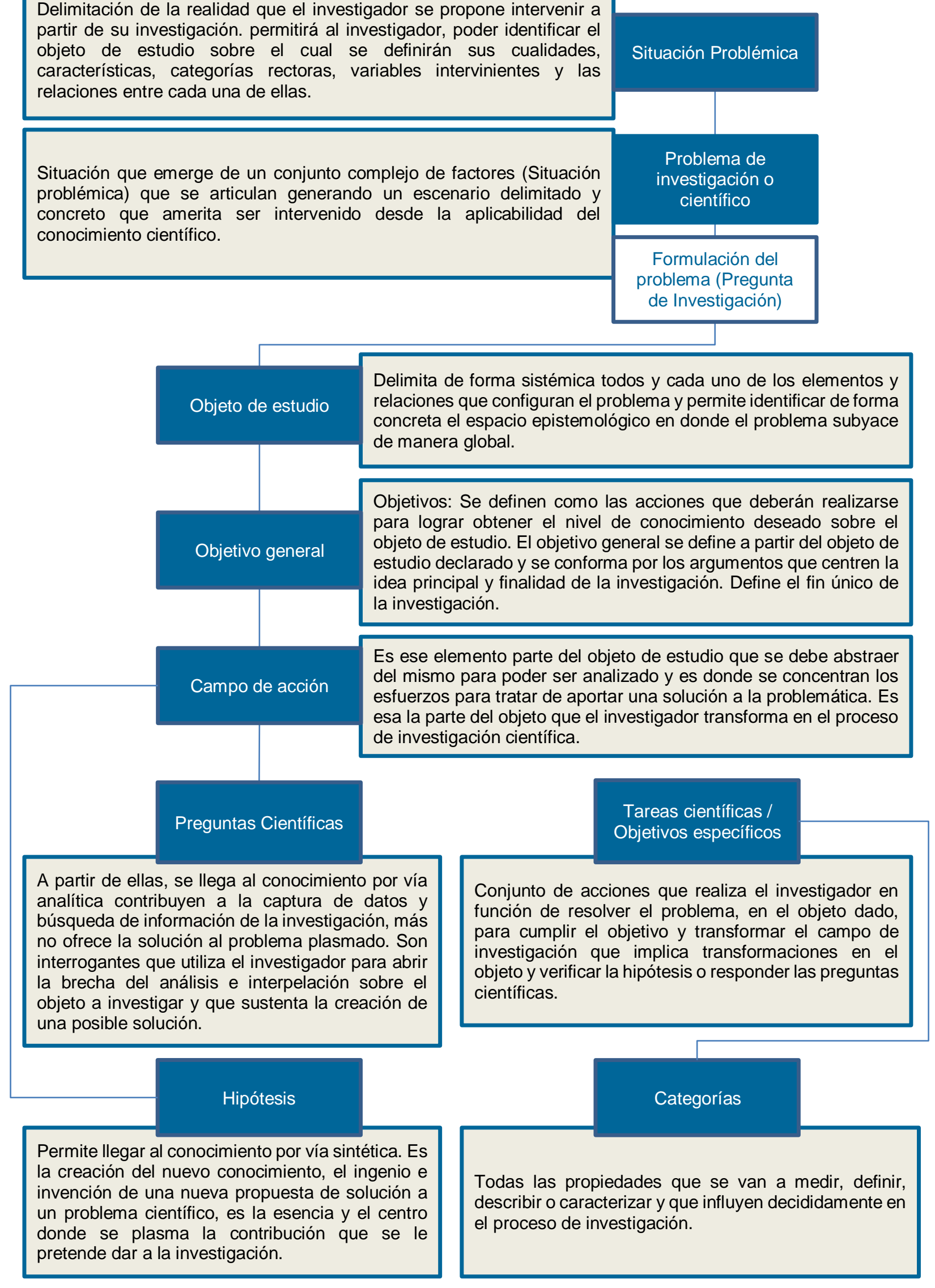

Fig. 1: Esquema propuesto para el diseño teórico de la investigación 


\section{Situación Problémica / Realidad problémica}

Podría entenderse como la delimitación de la realidad que el investigador se propone intervenir a partir de su investigación. La situación problémica permitirá al investigador, poder identificar el objeto de estudio sobre el cual se definirán sus cualidades, características, categorías rectoras, variables intervinientes y las relaciones entre cada una de ellas. En términos sencillos, la situación problémica pudiera resumirse en el conjunto de factores o circunstancias que afectan a algo o a alguien (objeto de estudio), es decir la serie de eventos y elementos asociados a un determinado objeto que genera en el investigador la necesidad de analizarlo de manera crítica y detallada. Diversos autores coinciden en afirmar que la situación problémica se puede entender como una situación caótica que general un problema, sin embargo, no siempre la situación problémica es sinónimo de caos o descontrol, por el contrario, podría concretarse como una situación que a juicio de un observador merece una atención especial teniendo en cuenta el contexto en donde la misma se desarrolle.

\section{Problema de investigación o problema científico}

El problema en términos de investigación podría definirse como una situación que emerge de un conjunto complejo de factores (Situación problémica) que se articulan generando un escenario delimitado y concreto que amerita ser intervenido desde la aplicabilidad del conocimiento científico. Puede entenderse como una contradicción entre el estado actual y el estado ideal de una situación desde lo teórico o lo práctico, en donde se evidencia la inconformidad o la necesidad de intervenir a partir de un proyecto de investigación, por tanto, esta situación deberá delimitarse en algo más concreto, esa concreción configura el problema de investigación. Se deberá tener en cuenta que los problemas que se identifiquen, tendrán que estar lo suficientemente bien descritos, de forma tal que se favorezca su formulación a partir de la pregunta de investigación. Generalmente, los problemas de investigación / problemas sociales, conducen a estudios diagnósticos o exploratorios que exigen de la aplicación de los métodos de investigación científica, sus orientaciones por preguntas científicas permiten obtener y aportar informaciones y datos valiosos; conocimientos rigurosos y científicos, relacionados con el objeto de estudio. Los problemas científicos por su dinámica transformadora requieren una cientificidad en el método usado para su intervención, lo que favorece definir los aportes y/o soluciones desde lo teórico, practico y metodológico (Mackenzie y Knipe, 2006; Arksey y O’Malley, 2005).

\section{Formulación del problema de investigación}

La formulación del problema de investigación se asume como una de las fases más importantes del proceso investigativo, la misma consiste en definir de forma estructurada y sistémica, la idea central de la investigación que se propone realizar. En esta etapa, se concreta el objeto de estudio, definiéndose el campo de acción, es decir, esa parte del objeto que se pretende intervenir con la investigación y define la delimitación del proceso investigativo y su alcance. Hurtado (2014) define este proceso de formulación del problema como el asunto específico que se pretende investigar, es decir, es el proceso que define el cómo poder atender una situación que genera una necesidad y requiere ser intervenida a partir de la aplicación de un método de ciencia por parte del investigador. La formulación del problema deberá presentarse en forma de pregunta y esta, deberá ser precisa y sin vincular elementos, categorías o expresiones que se sugieran en su redacción una posible solución. Autores relevantes coinciden en afirmar que existen diferentes tipos de preguntas de investigación, el tipo que se defina para el proyecto, orienta el tipo de investigación que necesita realizar, al mismo tiempo, concreta el objetivo general de la investigación que al final determina y concreta el tipo de investigación y el o los métodos que se requieren y las técnicas asociadas a los mismos. Desde el enfoque holístico propuesto por Hurtado (2014), las categorías conceptuales definidas en las fases previas, por lo general, se relacionan de forma sistémica, permitiendo que una pregunta pueda combinar varios tipos.

\section{Objeto de estudio}

Se origina a partir del problema científico o problema de investigación de acuerdo con la situación analizada. Este se desarrolla en función del problema y por procesos, sin embargo, no es recomendable abarcar todos los componentes que integran la posible solución, sino, enfatizar sobre un campo de acción. El resultado del objeto de estudio y de su respectiva investigación, no consiste en tocar el planteamiento de diferentes puntos de vista, sino, de la precisión y la calidad con la cual se responde a la raíz del problema de investigación. En síntesis, al identificar y definir el objeto de estudio de la investigación, se delimitan de forma sistémica todos y cada uno de los elementos y relaciones que configuran el problema y permite identificar de forma concreta el espacio epistemológico en donde el problema subyace de manera global.

\section{Objetivo general}

El objetivo general se define a partir del objeto de estudio declarado y se conforma por los argumentos que centren la idea principal y finalidad de la investigación. Define el fin único de la investigación. La redacción del 
objetivo deberá iniciar con un verbo (infinitivo) que permita evaluar el alcance del resultado esperado, de lo contrario, su planteamiento conformará parte de otro texto complementario. En algunos casos, el objetivo conlleva a concretar el campo de acción y a la definición del título final del trabajo de investigación. Finalmente, la definición y formulación del objetivo, precisa el tipo de investigación que se pretende llevar a cabo.

\section{Campo de acción}

Componente que se determina en el objetivo general de la investigación y se define en el objeto de estudio. El campo de acción, si bien hace parte de la situación problémica que se desea investigar, éste se plasma en el objetivo general; es aquí donde el campo de acción se plantea a partir una particularidad, cualidad o componente inherente al objeto de estudio. El investigador presupone que al delimitar el campo de acción se podría lograr el objetivo mencionado. El campo de acción puede entenderse de forma sencilla como una concreción del objeto de estudio, es ese elemento parte del objeto que se debe abstraer del mismo para poder ser analizado y es donde se concentran los esfuerzos para tratar de aportar una solución a la problemática. Es esa la parte del objeto que el investigador transforma en el proceso de investigación científica.

\section{Preguntas Científicas}

Promueven la dinámica del conocimiento con el objetivo de conocer y comprender la realidad contradictoria de la investigación. Según sea el campo de acción, responde a los "vacíos" que dan origen al planteamiento sujeto-investigador en su contexto científico y su cultura profesional (Hamui, 2015). Responden a cuestionamientos que fundamentan la investigación en la búsqueda exploratoria de información. Las preguntas científicas argumentan tanto los efectos como las causas de su razón de ser y se manifiestan desde lo no propositivo a lo propositivo, éstas se presentan y se revelan en el transcurso de la investigación en cualquier momento que se considere pertinente. De esta manera, la pregunta científica es quien soporta la situación problémica y demuestra la pertinencia de un resultado científico propuesto. No existe un número mínimo o máximo de preguntas, será el investigador quien defina estos criterios de acuerdo con la dinámica misma de su proceso investigativo. Una pregunta científica, podrá tener una o varias tareas asociadas para su posible respuesta. En general, la pregunta científica contribuye a la captura de datos y búsqueda de información de la investigación, más no ofrece la solución al problema plasmado. Son interrogantes que utiliza el investigador para abrir la brecha del análisis e interpelación sobre el objeto a investigar y que sustenta la creación de una posible solución (Browne y Keeley, 2018).

\section{Tareas científicas / Objetivos específicos}

Son las definiciones particulares de la declaración operacional del objetivo general. Su formulación consta de diferentes enunciados que incluyen dimensiones e indicadores en función del cumplimiento del objetivo general. Por tanto, los objetivos específicos son la parte más clara y precisa que se describe del alcance y del resultado esperado, es importante aclarar, que se realizan luego de haber proyectado el objetivo general.

En resumen, se puede concluir que la metodología que se ofrece para el diseño teórico de la investigación científica y su fundamentación corresponde a una lógica interna de la investigación; cabe aclarar, que su formulación y dinamismo entre las partes están integradas entre sí, son flexibles y no pierden su sentido creativo para la toma de decisión según sea el caso. Es importante tener en cuenta que, en el proceso de desarrollo del diseño teórico, el resultado que concluye cada uno de sus componentes en su formulación, sirve de entrada al siguiente, es decir, que en la transición de un componente a otro existe una retroalimentación que apoya la razón de ser de la investigación.

\section{Hipótesis}

Sobre este elemento del diseño teórico, muchos autores coinciden de manera general que una hipótesis puede ser entendida como es una expresión conjetural que se evidencia a partir de la relación existente entre dos o más variables, que además debe ser verificable a través de la experiencia, indistintamente cual sea la definición que acepte, se hace necesario precisar algunos aspectos que pueden ser relevantes al momento de definir su incorporación o no en un proyecto de investigación.

En primer lugar, en muchas investigaciones es posible identificar el concepto de hipótesis como equivalente a una idea central o tesis a defender, en ese sentido, se precisa que, para defender una idea central o tesis, se requiere de una lógica estructurada de razonamiento y no de un proceso investigativo propiamente tal. En segundo lugar, tal como se expone al inicio, las hipótesis se formulan en investigaciones donde se definen relaciones causa efecto que se pretenden verificar, específicamente las del tipo confirmatorio y evaluativo. Por ejemplo: Cuando un investigador formula una pregunta de tipo descriptivo, es porque no se conocen las características del evento en contexto de este y bajo esas condiciones, no tendría razón de ser afirmar 
anticipadamente lo que se podría encontrar, cuando ni siquiera se tienen indicios al respecto. En cambio, cuando se plantea una hipótesis en un tipo de investigación confirmatoria, por ejemplo, es porque se tiene certeza sobre la existencia de teorías previas que sustentan la misma. En el evento en que por el tipo de investigación se requiera su inclusión, esta deberá estar orientada y alineada al campo de acción, por tanto, deberá formularse después de definirlo y luego de haber concretado las distintas respuestas a las preguntas científicas enunciadas.

En síntesis, en el espiral holístico de investigación propuesto por Hurtado (2014), en los primeros tipos de investigación (exploratoria, descriptiva, analítica y comparativa) no se formulan hipótesis en función de que en las mismas no se definen con relaciones de causa y efecto. Finalmente, para los efectos de mantener la dinámica entre los diferentes elementos del diseño teórico, se incorpora la hipótesis para ser tenida en cuenta a discreción del investigador en consecuencia de las precisiones antes descritas.

\section{Categorías}

Las categorías se consideran un elemento fundamental del diseño desde lo teórico y la dinámica misma del proceso. Las mismas responden a la necesidad de poder definir una serie de elementos de tipo conceptual que a manera de palabras clave o estructuras teóricas permitan estructurar de forma articulada los distintos referentes para organizar de forma eficiente la información que se requiere. Las categorías son identificadas y definidas desde la situación problémica, puesto que las mismas están inmersas en el problema mismo y en sus diferentes dimensiones de aplicación.

\section{RESULTADOS Y DISCUSIÓN}

A continuación, se presenta la operacionalización metodológica del esquema de diseño teórico de la investigación aquí propuesto a través del desarrollo de dos (2) casos modelo, respectivamente representados en las figuras 2 y 3. La figura 2 corresponde al Caso 1: Gestión de conocimiento en empresas del sector TI, y la figura 3 al Caso 2: Didáctica para la resolución de algoritmos en el programa de ingeniería de sistemas (Londoño y Sánchez, 2015).

El diseño teórico de la investigación se define como una metodología de trabajo investigativo que permite orientar una investigación desde las necesidades del entorno, permitiendo articular una serie de elementos necesarios con el objetivo de en primera instancia, poder identificar lo que genera la necesidad de investigar y luego describir las etapas que se necesitan definir para encontrar la solución a una necesidad. En la actualidad, los elementos componentes del diseño teórico de la investigación científica, son tratados de distintas formas y articulados de acuerdo con la visión particular de cada investigador (llámese estudiante, profesor o profesionales de cualquier rama del saber), en donde cada uno propone y diseña el camino que mejor considere para llegar a un resultado que desde la ciencia avale su implementación.

Los componentes diseño teórico de la investigación (DTI), están sistémicamente articulados por vínculos conceptuales, lógicos y formales que son los elementos rectores de toda investigación, determinando en secuencia la proyección metodológica posterior en cuanto a la selección de la muestra, los métodos, las técnicas y los procedimientos. Son al mismo tiempo, el punto de referencia para el procesamiento de los resultados y el establecimiento de las correspondientes conclusiones y recomendaciones.

Es posible que, en dependencia del nivel de formación profesional, se trate de delimitar el alcance de los elementos y dinámica del diseño teórico de la investigación, incluso se sugiera un nivel de asimilación y de profundidad del diseño teórico dependiendo del nivel. El diseño teórico se define como único, independientemente del nivel de formación en el que se aplique, es decir, tiene la misma rigurosidad, dinámica, exigencia y sistematicidad, tanto para los niveles de formación de pregrado y postgrado. Lo anterior presupone que cada diseño teórico deberá corresponderse con una situación problémica determinada, con características y particularidades que la hacen única y que el nivel de exigencia formativa distinto al propuesto de manera inicial.

El diseño teórico de la investigación científica sugiere en su complejidad no ser ajustado a procesos diferentes al desarrollo de propuestas y proyectos de investigación científica en toda su generalidad. Su lógica interna como expresión metodológica de conformación y estructura, puede entenderse como universal, es decir común a todas las ciencias indistintamente de los problemas que en cada una se profundicen o se pretendan intervenir. Son ellos, los problemas, los que definen el uso triangulado de los paradigmas de investigación que, para este caso en particular, dejaría de ser un motivo de preocupación para el investigador pues, la concepción holística de esta propuesta desecha ese grupo de restricciones que desde lo epistémico pudieran generar conflictos en los resultados finales del proceso investigativo (Guardo, 2009). 
Una buena parte de los fallos que se producen en el seno de las organizaciones proceden de una falta de comunicación y retroalimentación entre los diferentes departamentos y/o profesionales. Para minimizarlos, surge la gestión del conocimiento en las empresas como mecanismo para expandir la información relevante por toda la compañía.

Empresas de élite como IBM, Microsoft o Ernst \& Young, entre muchas otras, han comenzado a implementar estrategias en la gestión de conocimiento que les permitan aumentar su productividad y competitividad...

Bajos índices de aplicabilidad de procesos de gestión de conocimiento en las pymes del sector TI en la Región Caribe colombiana.

¿Cómo mejorar los procesos organizacionales en las empresas del sector TI de la Región

Caribe colombiana?

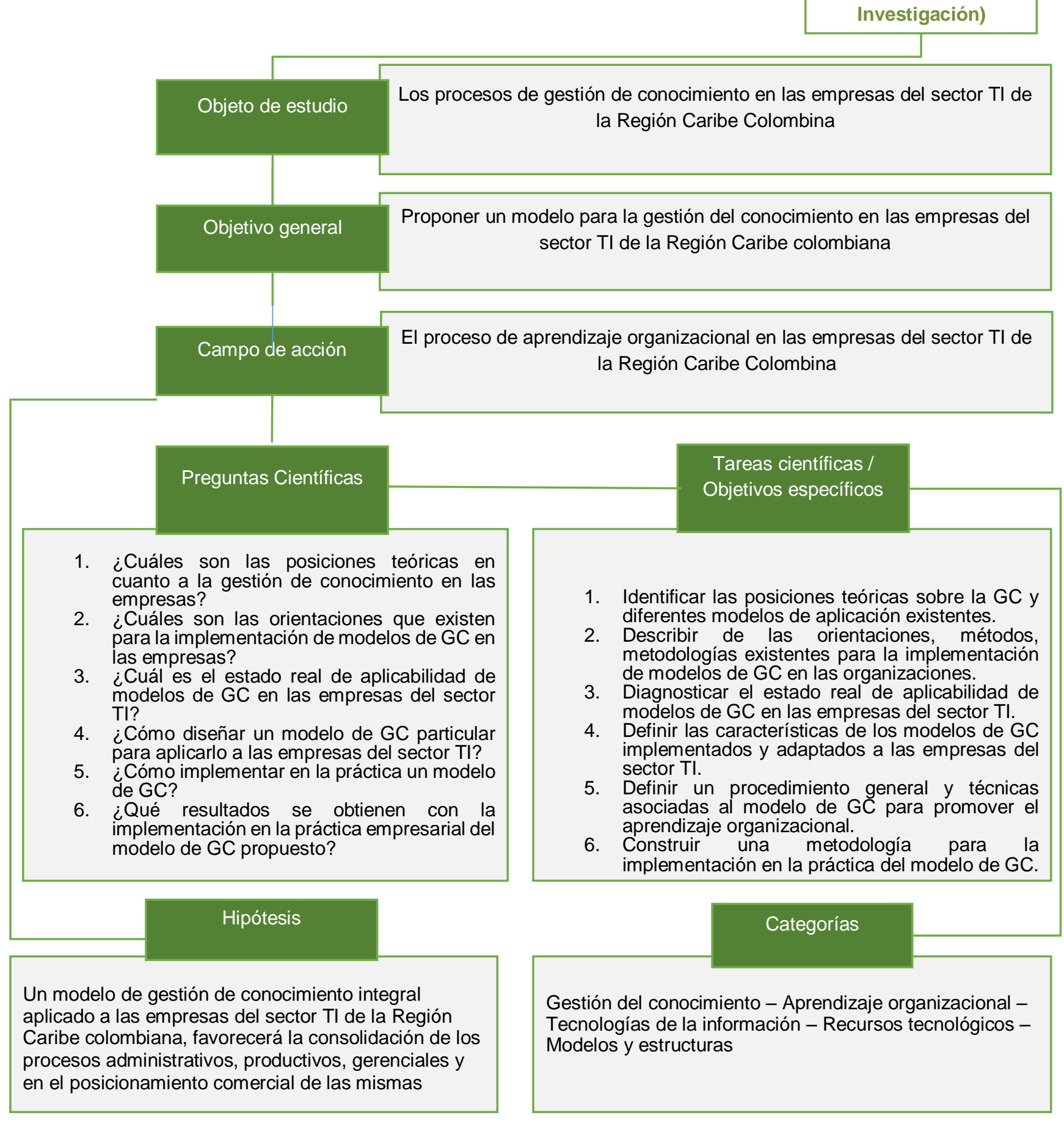

Fig. 2: DTI de Caso 1_Gestión de conocimiento en empresas del sector TI 
La gran mayoría de los estudiantes del programa de ingeniería de sistemas, a pesar de tener las competencias y aptitudes requeridas para cursar dicho programa, su desarrollo académico frente a uno de los cursos más relevantes de su ciclo profesional, no es tan bueno. Lo anterior referido a los bajos niveles en las calificaciones asociadas con el grado de apropiación de las metodologías asociadas con el aprendizaje de los algoritmos y su aplicabilidad en situaciones profesionales reales y simuladas de su carrera al interior de los cursos en los que se imparten estos contenidos...

Resultados académicos poco satisfactorios de los estudiantes del programa de ingeniería de sistemas en la resolución de algoritmos en el curso de programación I.

¿Cómo favorecer el aprendizaje de la resolución de algoritmos en los estudiantes del curso programación I del programa de ingeniería de sistemas?

Problema de

investigación

Formulación (Pregunta de Investigación)

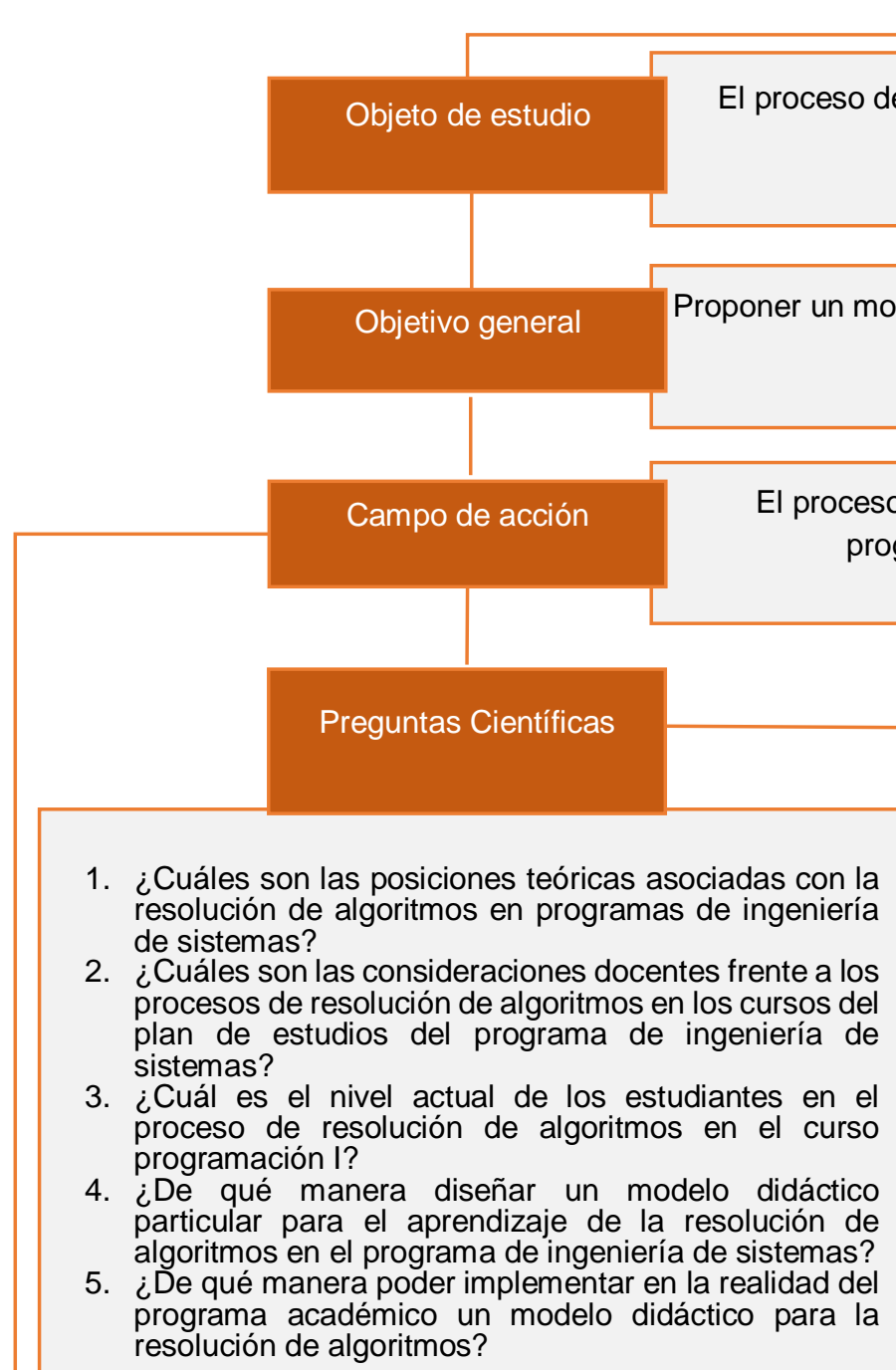

Hipótesis

Un modelo didáctico favorecerá la apropiación de herramientas teórico-metodológicas para fomentar el aprendizaje de la resolución de algoritmos en los estudiantes del programa de ingeniería de sistemas.
Tareas científicas /

Objetivos específicos
1. Identificar las posiciones teóricas sobre la resolución de algoritmos en los programas de ingeniería de sistemas.

2. Describir de las orientaciones, métodos, metodologías utilizadas por los docentes en el proceso de enseñanza aprendizaje de la resolución de algoritmos.

3. Diagnosticar el nivel real de apropiación de conceptos y dominio de las metodologías para la resolución de algoritmos en el programa de ingeniería de sistemas.

4. Definir las características y particularidades de los modelos y sus respectivas derivaciones entre pedagógicos y didácticos.

5. Definir una metodología con sus respectivas indicaciones de aplicabilidad para el aprendizaje de la resolución de algoritmos en el programa de ingeniería de sistemas.

Fig. 3: DTI de Caso 2_ Didáctica para la resolución de algoritmos en el programa de ingeniería de sistemas 
Dicho lo anterior, es el diseño teórico el que determina el nivel de profundidad en que pueda aplicarse o no un paradigma con relación a otro en el proceso de investigación, lo que se corresponde con lo dicho en apartes anteriores que pueden no existir investigaciones que respondan a un paradigma único, sino que existe entre ellos un nivel de complementariedad a menor y mayor nivel según sea el caso. En este contexto, la investigación holística se presenta como una alternativa metodológica que hace posible formular propuestas de investigación, facilitando el trabajo de estructurar un diseño lógico, coherente, sistémico que en su dinámica contribuya con la generación de una nueva cultura investigativa, más sencilla sin dejar de ser rigurosa en su cientificidad. Este tipo de investigación se presenta como un sintagma de los diferentes modelos epistémicos; se concibe como un proceso global, evolutivo, integrador, concatenado y sinérgico, con aspectos secuenciales y simultáneos, lo que facilita el proceso de enseñanza aprendizaje de la metodología de la investigación científica como curso en los diferentes niveles de la educación superior.

En el marco de la investigación holística, el enfoque de sistemas se constituye en una orientación metodológica dirigida a modelar el objeto mediante la determinación de sus componentes, así como las relaciones entre ellos, que conforman una nueva cualidad como totalidad, esas relaciones determinan por un lado la estructura y la jerarquía de cada componente en el objeto y por otra parte su dinámica y su funcionamiento. Lo anterior puede entenderse como una metodología que permite la intervención en una situación profesional considerada y entendida como un sistema (Objeto de estudio) y en las relaciones que ella tiene con su contexto, lo anterior presupone la descripción de la situación problémica, explicación de los objetivos, identificación de uno o varios puntos críticos, determinación del modo de intervención, elaboración de las estrategias que tengan en cuenta las interacciones conocidas entre las instituciones, participantes y el contexto social (Palomo, et al., 2008).

\section{DISCUSIÓN FINAL}

La propuesta metodológica, se fundamenta teóricamente en los elementos que a manera de categorías didácticas y pedagógicas son asumidos desde la concepción crítica de aportes semánticos y metodológicos de la teoría general de sistemas y los elementos estructurales del proceso de investigación científica. Los aportes metodológicos y de aplicación de este trabajo se definieron a partir de la relación didáctica entre el análisis con enfoque sistémico y el tratamiento dinámico de situaciones profesionales, estas últimas, consideradas como aquellas en las que, desde el punto de vista del investigador y sus necesidades, pueden abordarse desde diferentes enfoques, permitiendo su entender y tratamiento desde la comprensión holística de la ciencia.

El esquema metodológico (DTI) propuesto en este artículo, representa teóricamente la dinámica del proceso investigativo desde una visión sencilla y acorde con los nuevos enfoques y diferentes marcos epistémicos de investigación científica dispuestos en la actualidad, así como los procesos de transposición didáctica de situaciones profesionales, desde una perspectiva holística, la cual surge como respuesta a la necesidad de integrar los distintos enfoques, métodos y técnicas, que desde las diversas disciplinas, han permeado el desarrollo del conocimiento científico, favoreciendo los procesos de instrucción, desarrollo y educación en materia investigativa. Lo anterior, referido a la importancia de definir objetivos claros para la investigación, los cuales se entienden como los logros sucesivos de procesos continuos más que como un resultado final. El fijarse en los objetivos hace que los paradigmas de la investigación desaparezcan (Hurtado, 2014).

En términos del enfoque holístico de investigación, este implica la integración todos los espectros y paradigmas de investigación cuantitativos y cualitativos. Ello incluye emociones, conductas, percepciones, cosmovisión, entre otros, lo cual cobra una importante relevancia científica hoy en día, producto del caos social y las manifestaciones ideológicas y comportamentales en las diversas culturas. En el mismo sentido, esta visión integradora y sistémica, pudiera causar algún tipo de resistencia en quienes tradicionalmente apoyan la visión positivista, bajo el entendido que los primeros desarrollos metodológicos se hicieron en el marco de ese enfoque, fortaleciendo la idea de confirmar hipótesis, validando como único el método científico tradicional y resistiendo a cualquier otra manera de hacer ciencia. Finalmente, en correspondencia con lo propuesto por García-González y Sánchez-Sánchez (2017), se presenta una transposición didáctica entre los diferentes elementos del DTI y los diferentes enfoques metodológicos aplicados para el diseño de investigaciones.

\section{CONCLUSIONES}

De acuerdo al trabajo presentado y a los resultados obtenidos, se pueden plantear las siguientes conclusiones principales:

1.- El tratamiento dinámico de situaciones profesionales son consideradas como aquellas en las que, desde el punto de vista del investigador y sus necesidades, pueden abordarse desde diferentes enfoques, permitiendo su entender y tratamiento desde la comprensión holística de la ciencia. 
2.- El esquema metodológico (DTI) propuesto en este artículo, representa teóricamente la dinámica del proceso investigativo desde una visión sencilla y acorde con los nuevos enfoques y diferentes marcos epistémicos de investigación científica dispuestos en la actualidad.

3.- Esta visión integradora y sistémica, pudiera causar algún tipo de resistencia en quienes tradicionalmente apoyan la visión positivista, bajo el entendido que los primeros desarrollos metodológicos se hicieron en el marco de ese enfoque, fortaleciendo la idea de confirmar hipótesis, validando como único el método científico tradicional y resistiendo a cualquier otra manera de hacer ciencia.

\section{REFERENCIAS}

Arksey, H. y O'Malley, L., Scoping studies: towards a methodological framework, dx.doi.org/10.1080/1364557032000119616, Int J Soc Res Methodol 8, 19-32 (2005).

Bayazit, N., Investigating design: a review of forty years of design research, doi.org/10.1162/074793604772933739, Design Issues, 20(1), 16-29 (2004).

Browne, M.N. y Keeley, S., Asking the Right Questions: A Guide to Critical Thinking, 12th Ed, Pearson (2018).

Caillaud, E., Rose, B. y Goepp, V., Research methodology for systems engineering: some recommendations, doi.org/10.1016/j.ifacol.2016.07.803, IFAC-PapersOnLine, 49(12), 1567-1572 (2016).

Corona, J.L., Investigación cualitativa: fundamentos epistemológicos, teóricos y metodológicos, doi.org/10.15178/va.2018.144.69-76, Vivat Academia, 144, 69-76 (2018).

Davis, J., Mengersen, K., Bennett, S. y Mazerolle, L., Viewing systematic reviews and meta-analysis in social research through different lenses, doi.org/10.1186/2193-1801-3-511, SpringerPlus 3, 511 (2014).

García-González, G. y Sánchez-Sánchez, P.A., El Enfoque de Sistemas como Alternativa Didáctica del Horizonte SocioCrítico de la Universidad Simón Bolívar, en la gestión curricular como investigación para el desarrollo y fortalecimiento de los procesos de formación en las organizaciones educativas, pp. 259-277, Ed. Universidad Simón Bolívar, (2017).

García-González, J.R., Sánchez-Sánchez, P.A., Orozco, M. y Obredor, S., Extracción de conocimiento para la predicción y análisis de los resultados de la prueba de calidad de la educación superior en Colombia, dx.doi.org/10.4067/S071850062019000400055, Formación universitaria, 12(4), 55-62 (2019).

Génova, G. y González, M., Cuatro Problemas del Método Científico-Experimental que Reclaman la Apertura a la Inteligencia Meta-Metódica, in Inteligencia y Filosofía, by Orial, M., pp 661-680, Marova Ed., España (2012).

Gómez, Á., Epistemología y metodología de la investigación científica en la filosofía experimental de galileo galilei y francis bacon, doi.org/10.33539/consensus.2018.v23n1.1472, Consensus, 23(1), 9-16 (2019).

González, A. y Hernández, A., Positivismo, dialéctica materialista y fenomenología: tres enfoques filosóficos del método científico y la investigación educativa, doi.org/10.15517/AIE.V14I3.16155, Actualidades Investigativas en Educación, 14 (3), 1-20 (2014).

Grant, M.J. y Booth, A., A typology of reviews: an analysis of 14 review types and associated methodologies, doi.org/10.1111/j.1471-1842.2009.00848.x, Health Information \& Libraries Journal, 26, 91-108 (2009).

Guardo, M. Los componentes del diseño teórico de la investigación científica. una reflexión praxiológica, Pedagogía Universitaria, 14 (3), (2009).

Haddadi, A., Hosseini, A., Johansen, A. y Olsson, N., Pursuing value creation in construction by research a study of applied research methodologies, doi.org/10.1016/j.procs.2017.11.138, Procedia Computer Science, 121, 1080-1087 (2017).

Hamui, A., La pregunta de investigación en los estudios cualitativos, doi.org/10.1016/j.riem.2015.08.008, Investigación en Educación Médica, 5 (17), 49-54 (2015).

Hurtado, J., Metodología de la Investigación Holística. Guía para la compresión holística de la ciencia. 5aㅡ., Quirón Ed. Caracas, Venezuela, (2014).

Londoño, B. y Sánchez, P.A., Algoritmo novedoso para la detección de tareas repetitivas en el teclado, doi.org/10.17081/invinno.3.2.2031, Investig. innov. ing, vol. 3 (2), 55-69, (2015).

Mackenzie, N. y Knipe, S., Research dilemmas: paradigms, methods and methodology, Issues In Educational Research, 16(2), 193-205 (2006).

Marczyk, G.R., DeMatteo, D. y Festinger, D., Essentials of Research Design and Methodology, John Wiley \& Sons, New Jersey, USA, (2005).

Mertens, D.M., Philosophy in mixed methods teaching: the transformative paradigm as illustration, International Journal of Multiple Research Approaches, 4, 9-18 (2010).

Ortiz, E., Los niveles teóricos y metodológicos en la investigación educativa, doi.org/10.4067/S0717-554X2012000100002 Cinta de Moebio, 43, 14-23 (2012).

Palomo, I., Veloso, C. y Schmal, R., Sistema de gestión de la investigación en la universidad de Talca, Chile. dx.doi.org/10.4067/S0718-07642007000100014, Información tecnológica, 18(1), 97-106 (2007). 
Papachristos, G., A mechanism based transition research methodology: bridging analytical approaches, doi.org/10.1016/j.futures.2018.02.006, Futures, 98, 57-71 (2018).

Ramírez, F. y Zwerg-Villegas, A., Metodología de la investigación: más que una receta, AD-minister, 20, 91-111 (2012).

Saunders, M. y Bezzina, F., Reflections on conceptions of research methodology among management academics, doi.org/10.1016/j.emj.2015.06.002, European Management Journal, 33 (5), 297-304 (2015).

Sánchez-Sánchez, P.A, Zuluaga J.C., y otros cinco, Knowledge discovery in musical databases for moods detection, dx.doi.org/10.1109/TLA.2019.9011552, IEEE Lat Am Trans, 17(12), 2061-2068, (2019).

Schmitt, R., Systematic metaphor analysis as a method of qualitative research, Qualitative Report, 10(2), $358-394$ (2005).

Schmidt, J., Kafkas, S., y otros tres, Why are we learning this? using mixed methods to understand teachers' relevance statements and how they shape middle school students' perceptions of science utility, doi.org/10.1016/j.cedpsych.2018.08.005, Contemporary Educational Psychology, 57, 2019, Pages 9-31 (2019).

Snyder, H., Literature review as a research methodology: an overview and guidelines, doi.org/10.1016/j.jbusres.2019.07.039, Journal of Business Research, 104, 333-339 (2019).

Thomas, J.T. y Harden, A., Methods for the thematic synthesis of qualitative research in systematic reviews, doi.org/10.1186/1471-2288-8-45, BMC Med Res Methodol, 8, 45 (2008). 